\title{
Generación de electricidad a través de microorganismos en los sedimentos de Humedales de Ite-Tacna
}

\author{
Generation of electricity through microorganisms in Ite wetland sediments in \\ Tacna
}

\author{
${ }^{1}$ Carlos Tito Vargas \\ ${ }^{2}$ Daladier Castillo Cotrina
}

\section{RESUMEN}

En este trabajo se tuvo como objetivo diseñar y construir celdas de combustible microbianas (CCM), generar potenciales máximos de electricidad y establecer la curva de polarización a partir de muestras de sedimentos de los Humedales de Ite, Tacna, Perú. Se diseñó y construyó dos tipos de CCM. La primera fue de dos cámaras y la segunda de una. Ambas celdas fueron tipo batch. Los microorganismos utilizados fueron consorcios presentes en los sedimentos provenientes de los Humedales de Ite. Los resultados de generación de electricidad fueron $686 \mathrm{mV}$ para la CCM de dos cámaras con materia orgánica, $442 \mathrm{mV}$ para la CCM de una cámara con materia orgánica y de $202 \mathrm{mV}$ para la CMM de una cámara sin materia orgánica. Se obtuvo una curva de polarización relacionando la densidad de potencia con la densidad de corriente, comprobándose que se empezó a tener pérdidas por disminución de sustrato o transporte de masas a partir del día 11 para la CMM de dos cámaras, del día 12 para la CCM de una cámara con materia orgánica y del día 6 para la CCM de una cámara sin materia orgánica.

Palabras clave: Bioelectricidad, celdas de combustible microbiana, consorcio microbiano, curva de polarización.

\section{ABSTRACT}

The objective of this work was to design and build microbial fuel cells (CCM), to generate maximum electricity potentials and to establish the polarization curve from sediment samples from Ite wetlands in Tacna, Peru. Two types of CCM were designed and built. The first was a two- chamber cell and the second a one-chamber cell. Both cells were batch type. The microorganisms used were consortiums present in the sediments from the Ite wetlands. The results of electricity generation was $686 \mathrm{mV}$ for the CCM of twochamber with organic matter; $442 \mathrm{mV}$, for the one-chamber CCM with organic matter; and of $202 \mathrm{mV}$ for the one chamber CMM without organic matter. A polarization curve was obtained by relating the power density with the current density, verifying that losses began due to substrate reduction or mass transport from day 11 for the CMM of two chamber, from day 12 for the one-chamber CCM with organic matter and from day 6 for the one chamber CCM without organic matter.

Keywords: Bioelectricity, microbial fuel cells, microbial consortium, polarization curve.

Universidad Nacional Jorge Basadre Grohmann. Tacna-Perú. E-mail: ctitov@unjbg.edu.pe

${ }^{2}$ Universidad Nacional Jorge Basadre Grohmann. Tacna-Perú. E-mail: daladiercastillo@hotmail.com 


\section{INTRODUCCIÓN}

La búsqueda de nuevas fuentes de energía no convencionales tiene por objetivo resolver necesidades como el de la demanda de electricidad. Uno de los argumentos más importantes, en la búsqueda de energías alternativas, es la no utilización de los derivados del petróleo, ya que evitan la producción y disminuyen la generación de dióxido de carbono $\left(\mathrm{CO}_{2}\right)$ y su emanación a la atmosfera, que dan como resultado el calentamiento global. Una de las energías alternativas es la que se produce por el aprovechamiento del metabolismo realizado por microorganismos, al utilizar los desechos de biomasa como materia prima, transformándola en energía eléctrica para ser utilizada por el hombre (Saavedra, 2012).

Los microorganismos están presentes en todos los ambientes y, a través de su metabolismo, pueden generar electricidad a partir de sedimentos, para lo cual se requiere el diseño de celdas de combustible microbianas (CCM) que conste de dos compartimentos, uno para que contenga al ánodo y otro para el cátodo (Alzate-Gaviria, Fuentes, Alvarez y Sebastian, 2008). En el compartimento anódico, están los sedimentos que contienen a los microorganismos, los cuales forman una biopelícula sobre el ánodo, que contribuyen con el flujo de electrones hacia el cátodo y con lo cual se genera electricidad. Estos microorganismos conforman consorcios que participan en estos procesos. A través de investigaciones en ecología microbiana se puede reconocer las relaciones entre los miembros de estas comunidades que contribuyen a generar mayor energía que los cultivos puros (Romero, Vásquez y Lugo, 2012).

Los factores imprescindibles para que se lleve a cabo el proceso de generación de electricidad, a través de la CCM, son los consorcios de microorganismos y el inóculo con el que se va a trabajar proveniente de los sedimentos. La relación de estos microorganismos, por asociación o dependencia de dos o más tipos diferentes, combinan sus capacidades metabólicas para catalizar un sustrato que no puede ser degradado por alguno de ellos de manera independiente (Castillo y Tito, 2012).

Los Humedales de Ite están ubicados a $95 \mathrm{~km}$ de la ciudad de Tacna. Se llega a este lugar, yendo por la carretera de la Costanera en dirección de Tacna rumbo al Puerto de Ilo. Presenta diversidad en flora y fauna. Contiene sedimentos con diversidad de metales provenientes de la actividad minera en Toquepala, Tacna, Perú; además, albergan gran cantidad de microorganismos que podrían ser útiles para la generación de bioelectricidad.
En nuestro país es muy poco lo que se ha estudiado sobre bioelectricidad; sin embargo, en el extranjero se le viene dando enorme importancia. Las bacterias que producen electricidad tienen un fuerte potencial de utilización porque representan una nueva fuente de energía amigable con el ambiente. Las bacterias que poseen citocromos $\mathrm{C}$, proteínas de membrana externa, generan electricidad.

Estas moléculas intervienen en la comunicación entre la bacteria y los electrodos (funcionan como un material conductor aceptando electrones), optimizando la generación de corriente eléctrica (Busalmen, Esteve-Nuñez y Feliu, 2008). Respecto a dicha comunicación, los microorganismos presentan una gran versatilidad, tal como bacterias del género Geobacter que transfieren electrones fuera de la célula (flujo extracelular), lo que está relacionado con el citocromo C (Esteve-Nuñez, 2008). Estos microorganismos se alimentan y "respiran" metales para producir electricidad que puede utilizarse para la biorremediación. Estos microorganismos pueden presentar más de cien copias de estos citocromos en su genoma, y pueden transferir sus electrones, producto de su metabolismo, a través de sus pilis hacia los electrodos; los cuales pueden ser colonizados por los microorganismos (Juárez, Kim, Nevin, Olvera, Reguera, Lovley \& Methé) (Pirbadian, Barchinger, Leung, Byun, Jangir, Bouhenni, Reed, Romine, Saffarini, Shi, Gorby, Golbeck \& El-Naggar, 2014).

El objetivo de este trabajo fue diseñar y construir celdas de combustible microbianas (CCM), generar potenciales de electricidad máximos y establecer la curva de polarización a partir de muestras de sedimentos de los Humedales de Ite, Tacna, Perú.

\section{MATERIAL Y MÉTODOS}

\section{Diseño, construcción y preparación para funcionamiento de las $\mathbf{C} \mathbf{C M}$}

Se diseñó una CCM con material de PVC y otra CCM con vidrio; ambas CCM contuvieron los electrodos, uno catódico y otro anódico. La CCM de PVC contuvo dos cámaras separadas por una membrana de intercambio de protones (MIP) (Nafion de $0.127 \mathrm{~mm}$ de espesor), intercalado en una unión universal que unía ambas cámaras (Saavedra, 2012), y la de vidrio, una cámara (Logroño, Echevarría, Recalde y Graziani, 2015). 
La CCM de dos cámaras tuvo electrodos de carbón grafito, tanto en el ánodo como en el cátodo. Estos electrodos fueron barras cilíndricas compactas, conductivos, biocompatibles y químicamente estables a la solución del reactor. Las cámaras se cerraron con una tapa de material de PVC, conectadas a conductores externamente para el paso de electrones. En la cámara que hizo de ánodo, tuvo $250 \mathrm{ml}$ de medio propio de la zona de estudio; y en la cámara que hizo de cátodo, tuvo $250 \mathrm{ml}$ de agua destilada a la cual se le insufló aire a través del motor aireador. Esta CCM fue diseñada y construida en forma única para la experimentación $\left(\mathrm{CCM}_{2}\right)$.

La CCM de una cámara fue de forma cilíndrica, tuvo electrodos que hicieron de ánodo y cátodo; contenidas en un mismo compartimiento, donde el ánodo estuvo sumergido en el fondo y el cátodo sumergido a nivel de la superficie del líquido. Este tipo de CCM fue de dos gradientes de densidad, el de mayor densidad estuvo en el fondo (constituido por la muestra diluida a investigar) y el de menor densidad, en la superficie de la columna del líquido donde se colocaron respectivamente el ánodo y el cátodo. Esta CCM de una cámara fue diseñada y construida para la experimentación en un número de dos $\left(\mathrm{CCM}_{1}\right.$ y $\left.\mathrm{CCM}_{\mathrm{o}}\right)$.

\section{Muestreo y acondicionamiento para el funcionamiento de las CCM}

Se tomaron muestras de sedimento de los humedales de Ite, Tacna, Perú en frascos de vidrio de boca ancha y estériles, llenando los dos tercios del frasco, por triplicado, de un punto aledaño a la vegetación $\left(\mathrm{P}_{2}\right)$ a una profundidad de $80 \mathrm{~cm}$ y de un segundo punto $\left(\mathrm{P}_{1}\right)$ aledaño a la arena de playa a una profundidad de la superficie de $10 \mathrm{~cm}$ (DIGESA, 2007). Las muestras se conservaron en cajas térmicas "coolers" a temperatura de $4{ }^{\circ} \mathrm{C}$ y fueron trasladadas inmediatamente al laboratorio de Micología y Virología de la Facultad de Ciencias de la Universidad Nacional Jorge Basadre Grohmann. En el laboratorio las muestras fueron diluidas con agua destilada estéril en una proporción de 1:1.

La muestra del punto de muestreo $\mathrm{P}_{2}$ fue agregada a la cámara anódica de la $\mathrm{CCM}_{2}$ (Celda de combustible microbiana de dos cámaras con materia orgánica y aireación) en la cantidad de $250 \mathrm{ml}, \mathrm{pH}$ 6. Se agregó a la cámara catódica 250 $\mathrm{ml}$ de agua destilada. La cámara catódica fue insuflada con aire en un valor de $1 \mathrm{vvm}$ con un motor aireador. Las cámaras, anódica y catódica, se acondicionaron para que estuvieran conectadas externamente a través de un cable conductor eléctrico para que se generara electricidad. La incubación fue a $26^{\circ} \mathrm{C}$.

Se colocó $500 \mathrm{ml}$ de muestra, pH6, del punto de muestreo $\mathrm{P}_{2}$ al único compartimiento que tuvo la $\mathrm{CCM}_{1}$ (Celda de combustible microbiana de una celda con materia orgánica sin aireación). La muestra por diferencia de densidad formó una gradiente zonal constituida por una zona de mayor densidad ubicada en el fondo del recipiente (donde fue ubicado el electrodo ánodo) y una de menor densidad ubicada en la superficie de la muestra (donde fue ubicado el electrodo cátodo). La incubación fue a $26^{\circ} \mathrm{C}$ sin aireación.

Se colocó $500 \mathrm{ml}$ de muestra, pH 3.4, obtenido del punto de muestreo $\mathrm{P}_{1}$, al único compartimiento que tuvo la $\mathrm{CCM}_{\text {o }}$ (Celda de combustible microbiana de una celda sin materia orgánica ni aireación). A esta $\mathrm{CCM}_{\mathrm{o}}$ luego se le acondicionó y dejó que funcione como la $\mathrm{CCM}_{1}$.

\section{Mediciones en el funcionamiento de las CCM}

Se midió, diariamente, el potencial "E" $(\mathrm{mV})$ de corriente discontinua "DC" durante 19 días, utilizando un multímetro digital PRASEK Premium PR-75". Estas mediciones se hicieron de mayor a menor voltaje, para evitar dañar el equipo de medición.

Para la medición de la polarimetría, se registró datos diariamente usando resistencias, colocadas externamente a ambos cables externos de las CCM. Los valores de resistencia considerados fueron: 0 (sin colocar resistencia), 10, 20, 50, 200, 500, $1000,2000,10000,40000$ y 100000 ohmios.

En el cálculo de la intensidad de la corriente, I (Amperios) se utilizó la fórmula de la ley de $\mathrm{Ohms}$ $\left(\mathrm{I}=\mathrm{V} \cdot \mathrm{R}^{-1}\right)$, donde $\mathrm{V}=$ voltaje y $\mathrm{R}=$ resistencia. Para cada valor de potencia obtenida en la evaluación y de resistencia utilizada se obtuvo un valor de intensidad de corriente, I.

En la determinación de la densidad de corriente realizada, $j\left(\mathrm{~A} . \mathrm{m}^{-2}\right)$, se empleó la fórmula $j=\mathrm{IS}^{-1}, \mathrm{~S}$ (área en $\mathrm{m}^{2}$ del electrodo del ánodo), para cada valor de I calculado con los datos anteriores.

Se estableció la densidad de potencia, $\mathrm{Pd}\left(\mathrm{mW} \cdot \mathrm{m}^{-2}\right)$, empleando la fórmula $\mathrm{Pd}=\mathrm{IVS}^{-1}$ para cada valor de intensidad de corriente y de potencia obtenida. La curva de polarización obtenida se logró graficando la densidad de potencia versus la densidad de corriente para cada día. 


\section{Procesamiento y análisis de los datos}

Se utilizó el análisis de regresión y análisis de varianza a fin de establecer el grado de intensidad de asociación de las variables de estudio, tipo de celda utilizada y cantidad de energía generada. El coeficiente de correlación simple (R) permitió señalar la correlación existente entre ambas variables.

\section{RESULTADOS}

\section{En la CCM de dos cámaras con materia orgánica $\left(\mathrm{CCM}_{2}\right)$}

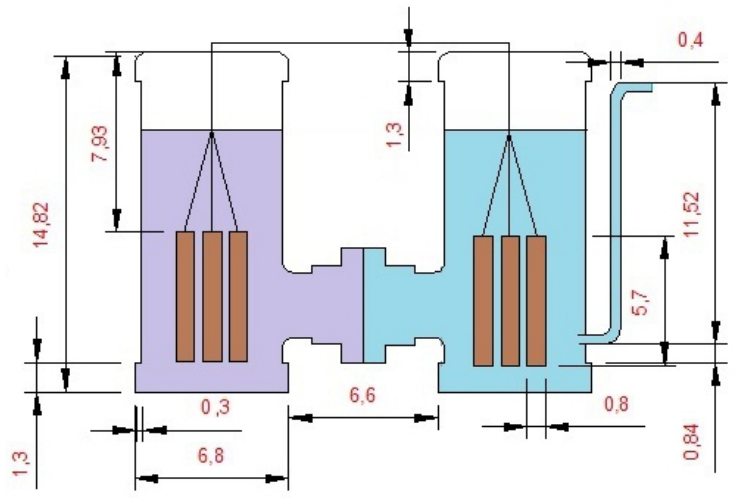

Figura 1. Diseño de CCM de dos cámaras con materia orgánica $\left(\mathrm{CCM}_{2}\right)$.

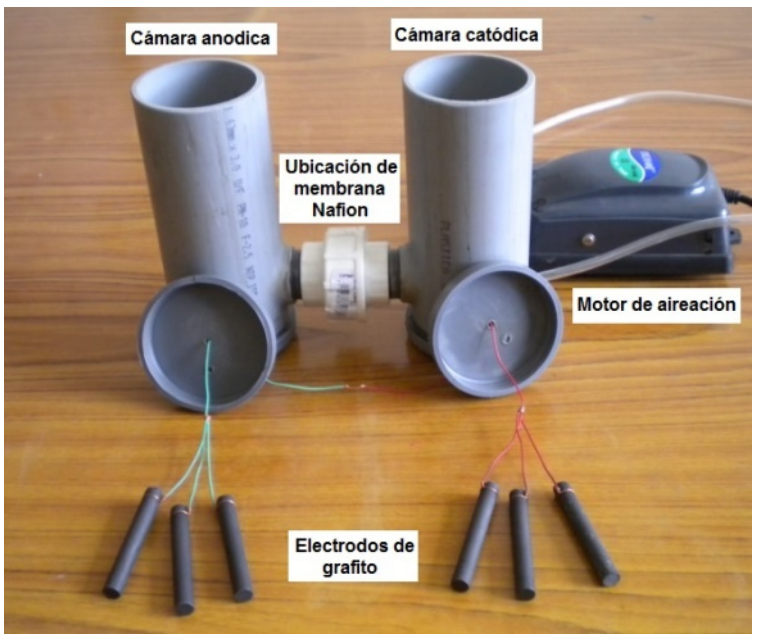

Figura 2. Componentes de las CCM de dos cámaras $\left(\mathrm{CCM}_{2}\right)$.

En la Figura 1 se muestra el diseño que sirvió para la construcción de la $\mathrm{CCM}_{2}$ (Figura 2).
En la CCM de una cámara $\left(\mathrm{CCM}_{0}\right.$ y $\left.\mathrm{CCM}_{1}\right)$

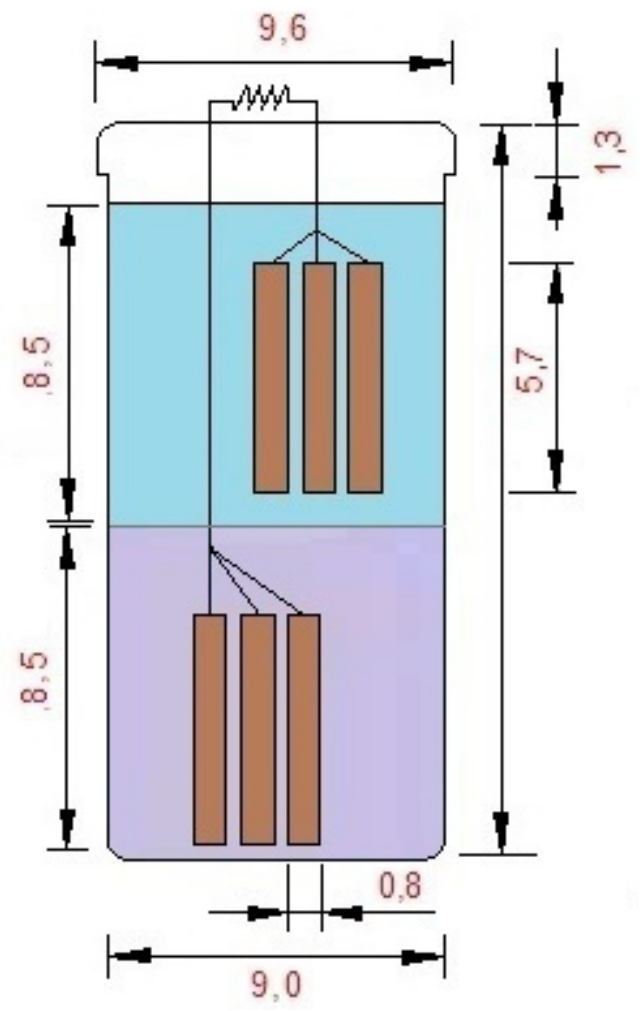

Figura 3. Diseño de la CCM de una cámara $\left(\mathrm{CCM}_{\circ}\right.$ y $\left.\mathrm{CCM}_{1}\right)$.

En la Figura 3 se muestra el diseño que se utilizó para la construcción de la celda de combustible microbiana de una celda $\left(\mathrm{CCM}_{1}\right.$ y $\left.\mathrm{CCM}_{\mathrm{o}}\right)$.

\section{Producción de electricidad en las CCM}

Tabla 1. Electricidad generada por la $\mathrm{CCM}_{2}$ (Celda de dos cámaras con materia orgánica y aireación), $C C M_{1}$ (Celda de una cámara con materia orgánica sin aireación) y $C C M_{o}$ (Celda de una cámara sin materia orgánica sin aireación)

$\begin{array}{cccc}\begin{array}{c}\text { Tiempo } \\ \text { (día) }\end{array} & \begin{array}{c}\mathrm{CCM}_{2} \\ \mathbf{m V}\end{array} & \begin{array}{c}\mathrm{CCM}_{\mathrm{o}} \\ \mathbf{m V}\end{array} & \begin{array}{c}\mathrm{CCM}_{1} \\ \mathbf{m V}\end{array} \\ 1 & 400 & 15 & 142 \\ 2 & 535 & 112 & 145 \\ 3 & 584 & 261 & 164 \\ 4 & 626 & 332 & 186 \\ 5 & 638 & 394 & 202 \\ 6 & 651 & 406 & 184 \\ 7 & 655 & 392 & 125 \\ 8 & 667 & 410 & 93\end{array}$




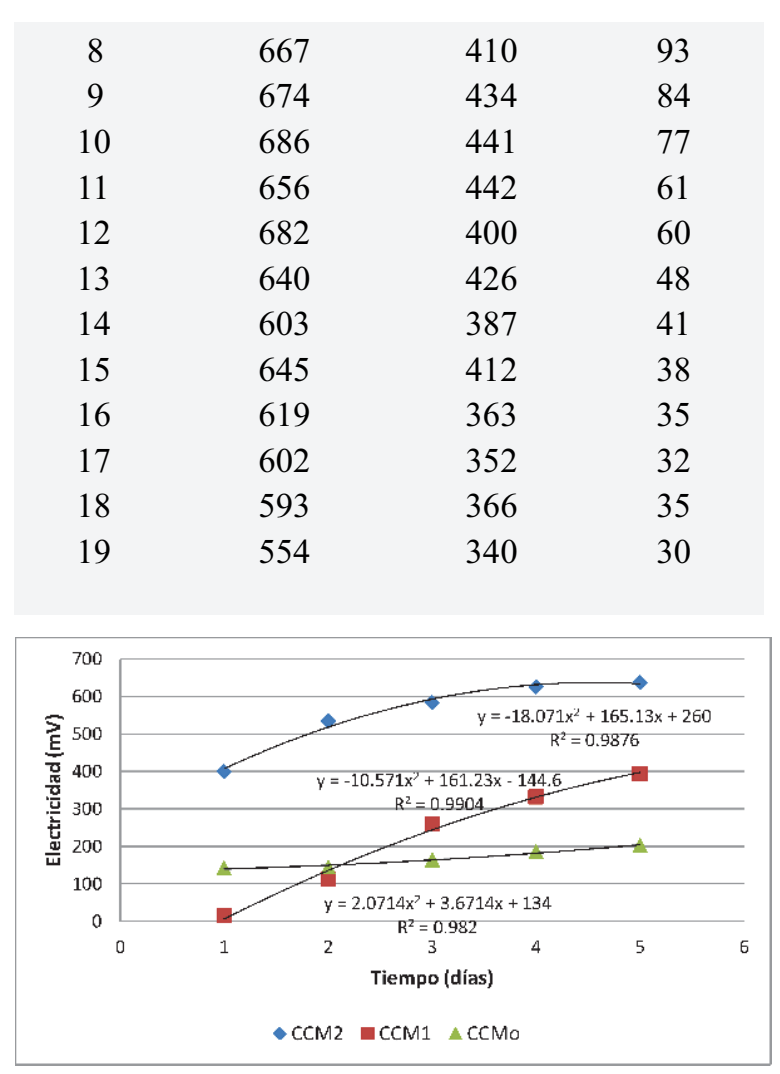

Figura 4. Curvas de electricidad obtenidas en $\mathrm{CCM}_{2}, \mathrm{CCM}_{1}$ $\mathrm{yCCM}_{\text {. }}$.

En la Tabla 1 se muestran los datos de electricidad $(\mathrm{mV})$ generados en las $\mathrm{CCM}_{2}, \mathrm{CCM}_{1}$ y $\mathrm{CCM}_{\mathrm{o}}$, donde se aprecia el mayor valor de $686 \mathrm{mV}$ a los 10 días de producción de electricidad en la $\mathrm{CCM}_{2}$. Estos datos de electricidad versus el tiempo de producción de electricidad pueden ser apreciadas en forma de curvas en la Figura 4.

\section{Análisis de regresión del tiempo y la generación de electricidad en la $\mathrm{CCM}_{2}, \mathrm{CCM}_{1} \mathrm{y}$ CCM}

Tabla 2. Grado de relación y correlación entre el tiempo y la generación de electricidad y su ecuación para cada $C C M$

$\begin{array}{cccc}\mathbf{C C M} & \mathbf{R} & \mathbf{R}^{2} \quad \text { Ecuación } \\ \mathbf{C C M}_{2} & 0.994 & 0.988 & \mathrm{Y}=-18.071 \mathrm{x}^{2}+165.13 \mathrm{x}+260 \\ \mathbf{C C M}_{1} & 0.995 & 0.990 & \mathrm{Y}=-10.571 \mathrm{x}^{2}+161.23 \mathrm{x}-144.6 \\ \mathbf{C C M}_{\mathbf{0}} & 0.991 & 0.982 & \mathrm{Y}=2.0714 \mathrm{x}^{2}+3.6714 \mathrm{x}+134\end{array}$

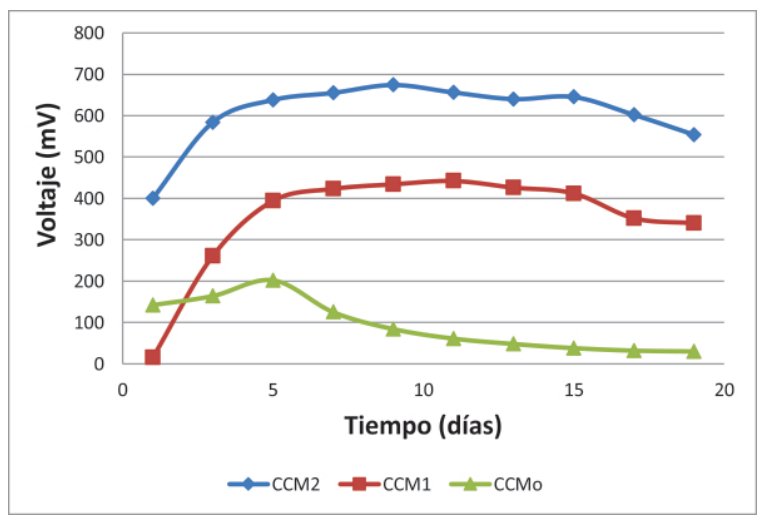

Figura 5. Curvas de análisis de regresión del tiempo y la generación de electricidad en la $\mathrm{CCM}_{2}, \mathrm{CCM}_{1}$ y CCM

En la Tabla 2 se aprecia la correlación entre el tiempo y la generación de electricidad en las tres celdas de combustibles trabajadas donde se evidencia la mayor correlación para la $\mathrm{CCM}_{1}$. Las respectivas curvas de análisis de regresión en las tres CCM se muestran en la Figura 5.

\section{DISCUSIÓN}

El uso de las celdas de combustible microbianas es una tecnología que busca nuevas fuentes de energía, utilizando el metabolismo de los microorganismos, por procesos catalíticos de óxido - reducción. En la CCM, el combustible (materia orgánica), alimentado en forma discontinua o batch a la cámara que contiene el ánodo, es oxidado biocatalíticamente durante el proceso metabólico de los microorganismos, donde finalmente los electrones producidos en esta cámara son transferidos al ánodo. Estos electrones, que están en este electrodo, migran hacia la segunda cámara que contiene el cátodo a través de los conductores externos, por la existencia de una diferencia de potencial. En la cámara catódica se produce una reducción bioquímica con la formación de agua. Los componentes de las CCM se han seleccionado basándose en sus propiedades y función que deben desempeñar. El ánodo debe tener gran área superficial, estabilidad química, biocompatiblilidad y buena conductividad. Aquí se produce la reacción de oxidación.

El ánodo cumple dos funciones importantes dentro de la celda de combustible microbiana: debe aceptar los electrones cedidos por las bacterias y deben transmitirlos al circuito externo. Esto implica que tiene que ser adecuado para el crecimiento bacteriano $\mathrm{y}$, sobre todo, para la colonización de los microorganismos formadores de biopelículas (biofilm). 
En cuanto al cátodo, este debe ser del mismo material que el ánodo, en este trabajo es el carbono; en la cámara que lo contiene se realiza una reacción abiótica, que consiste en una reducción del oxígeno y como consecuencia la formación de agua. La CCM de dos cámaras, las cuales están separadas por una membrana que permite la transferencia de carga (protones o $\mathrm{H}^{+}$), es una barrera que impide la mezcla de ambas soluciones (entre el combustible o anolito que contiene medio de cultivo en la cámara anódica; y el comburente o catolito que contiene agua destilada en la cámara catódica). Esta membrana de intercambio de protones, actúa como barrera física entre las soluciones y es permeable para que solamente los protones producidos en el ánodo puedan migrar hacia el cátodo y de esta manera mantener la electroneutralidad del sistema (Liu \& Logan, 2004). Este tipo de diseño presenta un circuito eléctrico externo situado entre el ánodo y el cátodo, consiste en una carga que introduce una resistencia al sistema. Este circuito debe estar bien aislado del entorno y asegurar una correcta conexión entre los electrodos, para tener buenas mediciones del sistema.

En la celda de una cámara o un solo compartimiento, esta hace las veces de zona anódica (en el fondo) y zona catódica (en la superficie). En este tipo de generador no existe una barrera física que permite una separación entre ambas zonas y los protones y electrones no tienen una eficiente migración de la zona anódica a la catódica, disminuyendo la producción de electricidad.

En cuanto a los mecanismos de medición de voltaje, son los mismos a los utilizados en la celda de dos cámaras, por lo que las lecturas se hicieron externamente con el multímetro en las terminaciones de los cables conductores ubicados en la única tapa del biorreactor.

En el proceso de generación de electricidad los voltajes máximos, expresados en potencial de 686 $\mathrm{mV}$ para la $\mathrm{CCM}_{2}$, de $442 \mathrm{mV}$ para la $\mathrm{CCM}_{1}$ y de $202 \mathrm{mV}$ para la $\mathrm{CCM}_{0}$, son mayores a los encontrados por Romero et al. (2012), Sharma \& Bulchandani (2012) y Castillo y Tito (2012); que obtuvieron valores de 200 a $300 \mathrm{mV}, 307 \mathrm{mV}$ y $151 \mathrm{mV}$, respectivamente. Valores cercanos se encontraron en los trabajos de Buitron y Pérez (2011); registrados desde $532 \mathrm{mV}$ a $660 \mathrm{mV}$. Sin embargo, en la mayoría de los casos los valores son relativamente bajos, los cuales pudieron deberse a las pérdidas óhmicas, por activación y por concentración (Saavedra, 2012) (Logan, 2009).

Según el análisis de regresión al obtenerse una mayor relación o asociación entre la cantidad de electricidad generada y el tiempo en la $\mathrm{CCM}_{1}, \mathrm{r}^{2}$ de 0.9904, seguido de la $\mathrm{CCM}_{2}, \mathrm{r}^{2}$ de 0.9876 y de la $\mathrm{CCM}_{o}, \mathrm{r}^{2}$ de 0.865 , se puede afirmar que en todos los casos existe un alto grado de correlación entre el tiempo y la generación de electricidad. En este trabajo se observa que en los tres tipos de CCM el p-valor es menor a 0.05 , lo que permite afirmar que las variables están linealmente relacionadas; Similares comportamientos fueron encontrados en los trabajos de Romero et al. (2012), Sharma \& Bulchandani (2011) y Castillo y Tito (2012).

\section{CONCLUSIONES}

Se diseñó y construyó dos tipos de CCM, la primera de dos cámaras cilíndricas tipo " $\mathrm{H}$ ", unidas por un conducto circular pequeño; en cuyo interior estuvo contenido una membrana de intercambio de $\mathrm{H}^{+}$que separó los contenidos de las cámaras; y la segunda de una cámara, de forma cilíndrica. En ambos casos de CCM, la construcción fue con material no conductor de electricidad.

La electricidad generada con un valor máximo fue de $686 \mathrm{mV}$ para la $\mathrm{CCM}_{2}, 442 \mathrm{mV}$ para la $\mathrm{CCM}_{1} \mathrm{y}$ de $202 \mathrm{mV}$ para la $\mathrm{CCM}_{0}$.

Se determinó una curva de polarización en la que a partir del día 15, se apreció pérdidas de electricidad por disminución de sustrato o transporte de masas en las $\mathrm{CCM}_{2}$ como en la $\mathrm{CCM}_{1}$.

\section{REFERENCIAS BIBLIOGRÁFICAS}

Alzate-Gaviria, I., Fuentes-Alabarrán, C., AlvarezGallegos, A. y Sebastian, P. (2008). Generación de electricidad a partir de una celda de combustible microbiana tipo PEM. Interciencia, 33(7), 503-509.

Buitrón, G. y Pérez, J. (2011). Producción de electricidad en celdas de combustible microbianas utilizando agua residual: efecto de la distancia entre electrodos. TIP Revista Especializada en Ciencias Químico-Biológicas, 14(1), 5-11. $\mathrm{R}$ e c u p e r a d o d e http://www.scielo.org.mx/pdf/tip/v14n1/v 14n1a1.pdf

Busalmen, J., Esteve-Nuñez, A. \& Feliu, J. (2008). Whole cell electrochemistry of electricity 
- producing microorganisms evidence an adapttation for optimal exocellular electron transport. Environmental. Science \& Technology, 42(7), 2445-2450. $\mathrm{R}$ e c u p e r a d o d e https://pubs.acs.org/doi/abs/10.1021/es70 $2569 \mathrm{y}$

Castillo, D., y Tito, C. (2012). Bioelectricidad generada en celdas de combustible microbiana a partir de diferentes substratos fermentados. RFN ${ }^{\circ}$ 73692013-FACI-UN/JBG. Tacna, Perú.

DIGESA. (2007). Protocolo de monitoreo de la calidad sanitaria de los recursos hídricos superficiales dirección de ecología y protección del ambiente área de protección de los recursos hídricos. Lima, Perú.

Esteve-Nuñez, A. (2008). Bacterias productoras de electricidad. Del sustrato a la pila de combustible. Actualidad. Recuperado de https://docplayer.es/12059754-Bacteriasproductoras-de-electricidad.html

Juárez, K., Kim, B., Nevin, K., Olvera, L., Reguera, G., Lovley, D. \& Methé, B. (2009). PilR, a Transcriptional Regulator for Pilin and Other Genes Required for Fe(III) Reduction in Geobacter sulfurreducens. Journal of Molecular Microbiology and Biotechnology, 16, 146-158. Recuperado de https://www.ncbi.nlm.ni.gov/pubmed/ 18253022

Liu, H. \& Logan, B. (2004). Electricity Generation Using an Air Cathode Single Chamber Microbial Fuel Cell in the Presence and Absence of a Proton Exchange Membrane. Environmental Science \& Technology, 38(14), 4040-4046. Recuperado de https://pubs.acs.org/doi/10.1021/es04993 44

Logan, B. (2009). Exoelectrogenic bacteria that power microbial fuel cells. Nature Reviews Microbiology, 7(5), 375-381. Recuperado de https://www.ncbi.nlm.nih.gov/pubmed /19d330018

Logroño, W., Echevarría, M., Recalde, C. y Graziani, P. (2015). Bioconversión de suelos de la región Amazónica y Alto Andina del Ecuador en celdas de combustible microbiano de cámara simple. Información tecnológica, 26(2), 61-68. Recuperado de https://scielo.conicyt.cl/ pdf/infotec/v26n2/art08.pdf

Pirbadian, S., Barchinger, S., Leung, K., Byun H., Jangir, Y., Bouhenni, R., Reed, S., Romine, M., Saffarini, D., Shi, L., Gorby, Y., Golbeck, J. \& El-Naggar, M. (2014). Shewanella oneidensis MR-1 nanowires are outer membrane and periplasmic extensions of the extracellular electron transport components. Proceedings of the National California Institute of Technology, 111(35), 12883-12888. Recuperado de https://www.pnas.org/co ntent/111/35/12883/tab-article-info

Romero, A., Vásquez, J. y Lugo. A. (2012). Bacterias, fuente de energía del futuro. Tecnura, 16(32), 118-143. Recuperado de http://www.scielo.org.co/pdf/tecn/v16n32 /v16n32a11.pdf

Saavedra, I. (2012). Diseño de una celda de combustible microbiológica con uso de bacterias oxidantes de azufre y hierro. Departamento de Ingeniería Química y Biotecnología. Facultad de Ciencias Físicas y Matemáticas. Universidad de Chile.

Sharma, S. \& Bulchandani B. (2012). Comparative Study of Various Substrates and Microorganisms in a Laboratory Designed Microbial Fuel Cell. International Journal of Research in Chemistry and Environment, 2 (3), 168-174. Recuperado de https://ijrce.org/uploads/20/833_pdf.p df. 